Kansas State University Libraries

New Prairie Press

\title{
VALIDITY OF 95\% t-CONFIDENCE INTERVALS UNDER SOME TRANSECT SAMPLING STRATEGIES
}

Stephen N. Sly

Jeffrey S. Pontius

James J. Higgins

Follow this and additional works at: https://newprairiepress.org/agstatconference

Part of the Agriculture Commons, and the Applied Statistics Commons

\section{(c) (1) $\Theta($}

This work is licensed under a Creative Commons Attribution-Noncommercial-No Derivative Works 4.0 License.

\section{Recommended Citation}

Sly, Stephen N.; Pontius, Jeffrey S.; and Higgins, James J. (1996). "VALIDITY OF 95\% t-CONFIDENCE INTERVALS UNDER SOME TRANSECT SAMPLING STRATEGIES," Conference on Applied Statistics in Agriculture. https://doi.org/10.4148/2475-7772.1325

This is brought to you for free and open access by the Conferences at New Prairie Press. It has been accepted for inclusion in Conference on Applied Statistics in Agriculture by an authorized administrator of New Prairie Press. For more information, please contact cads@k-state.edu. 


\title{
VALIDITY OF 95\% t-CONFIDENCE INTERVALS UNDER SOME TRANSECT SAMPLING STRATEGIES
}

\author{
Stephen N. Sly, Jeffrey S. Pontius, and James J. Higgins \\ Department of Statistics \\ Kansas State University
}

\begin{abstract}
Soil $\mathrm{pH}$ data were used to assess the capture rates of $95 \% \mathrm{t}$-confidence intervals based on five different transect sampling strategies. Two different sampling methods were considered, "deterministic" and "two-stage simple random sampling". The data used were $\mathrm{pH}$ readings at 15 and 30 centimeter depths from two local agricultural fields in the Manhattan, Kansas area. The data provided three distinct populations with three different distributions - skewed left, symmetric, and bimodal. The total number of transects randomly sampled was 2, 5, and 10 . The total number of points sampled along each transect was 2,7 and 14 . The $95 \%$ t-confidence intervals were simulated 5000 times using five different transect sampling strategies, and the capture rates of the population mean were recorded. Box plots of the capture rates for the five transect sampling strategies were constructed and compared. In most cases the "deterministic" sampling method had capture rates that underestimated the $95 \%$ confidence level, whereas the two-stage sampling strategies produced capture rates which were conservative or closer to the $95 \%$ confidence level. The variances of the capture rates for the two-stage sampling strategies were relatively small in comparison to the deterministic sampling strategies. In conclusion, the "two-stage simple random sampling" method along with the Satterthwaite degrees of freedom are recommended when using transect sampling.
\end{abstract}

\section{Introduction}

Researchers in agriculture and ecology quite often perform what is known as transect sampling. This method is accomplished by taking samples along straight lines which are called transects. One type of sampling along a transect is known as "deterministic" sampling, in which we would choose the locations to be sampled along each transect first, and then sample these fixed locations on every transect chosen. For example, agriculturists may take measurements of plants in a field by sampling every third plant within certain selected crop rows (transects). In practice, transects are either randomly selected or deterministically selected. When the locations are deterministically chosen each group of selected locations is a subpopulation. Therefore, the deterministic method may produce biased estimates if the actual population differs from the subpopulation of interest.

Our goals were to (a) compare two "deterministic" sampling strategies, (b) compare deterministic strategies with two-stage simple random sampling strategies, and (c) assess both deterministic and two-stage strategies under several empirical distributions of field data by simulating $500095 \%$ t-confidence intervals and recording the capture rates of the population mean for each strategy. In the first deterministic strategy, the transect means were treated as a simple random 
sample. In the second deterministic strategy all of the locations were treated as the random sample, which is quite often implemented by researchers. When performing two-stage simple random sampling without replacement (srswor), we randomly picked the transects and then also randomly picked the locations on each transect.

The degrees of freedom for the first and second methods of deterministic sampling using the Student t-distribution were $d f_{1}=\mathrm{MS}-1$ and $d f_{2}=\mathrm{MS} \cdot \mathrm{KS}-1$, respectively (MS = number of randomly selected transects; KS = number of randomly selected locations along each transect).

We found that it was very difficult to determine the proper degrees of freedom to use in the simulations for the t-confidence intervals under the two-stage simple random sampling method, especially because there is little information in the literature on construction of t-confidence intervals in finite population sampling. So similar degrees of freedom were also used in the two-stage simple random sampling method to provide a comparison with the deterministic results. The Satterthwaite degrees of freedom was our third choice and seemed reasonable for the two-stage srswor for two reasons (Satterthwaite, 1946). First, the Satterthwaite degrees of freedom is useful when two components of variation are present. It is useful when one level of variance $\left(\sigma_{2}{ }^{2}\right)$ is nested within another level of variance $\left(\sigma_{1}{ }^{2}\right)$ (Milliken and Johnson, 1992). Considering our transect data, there is variation across transects and then variation nested within each transect between the locations. Secondly, Satterthwaite degrees of freedom has been suggested in stratified random sampling (Cochran, 1977). Thinking of transects as strata provides a reasonable justification for using Satterthwaite degrees of freedom. Note that in stratified random sampling all of the strata are sampled, but under our sampling strategies only some of the transects are sampled. We performed transect sampling on three different data sets, which were obtained from the Agronomy Department at Kansas State University. Dr. John Havlin contributed the data, which were chemical compositions of soil samples taken from two local agricultural fields in the Manhattan, Kansas area.

\section{Methods}

Real data were used to assess the capture rates of t-confidence intervals based on five different transect sampling strategies. We define "sampling strategy" as the combination of a sampling method and an estimator ( $\mathrm{t}$-confidence interval). The data used were $\mathrm{pH}$ readings from two agricultural fields in the Manhattan, Kansas area. We shall refer to the two fields as the Phillips and Rice farms, which were named after the owners. The sample $\mathrm{pH}$ readings were taken at depths of 15 and 30 centimeters in the two fields.

\subsection{Sampling Grid}

In order to sample the data, a complete grid of 14 transects with 14 points along each transect was desired to have an equal number of locations along each transect. This 14 by 14 point grid is the population. However, the original data had not been sampled in a complete grid. The data in the corners of the grid were missing and extra $\mathrm{pH}$ readings were taken in the middle of the fields. Therefore, a Statistical Analysis System (SAS) program was written to complete a 14 by 14 grid. The procedure used in SAS (1985, version 6.10) was called PROC G3GRID, which utilized a fifth 
degree polynomial to interpolate the corners of the fields. The extra $\mathrm{pH}$ readings in the middle of each field were removed to have 14 transects and only 14 points along each transect. Contour maps before and after interpolating the corners verified that the pattern was maintained and that the added values (approximately 7\%) were a reasonable extension to the original grid values (Sly, 1996). The actual $\mathrm{pH}$ values for the three data sets after interpolating may also be referenced in Sly (1996).

We decided to use the Phillips field measured at both 15 and 30 centimeter depths, and the Rice field measured at a 15 centimeter depth only. We chose these three fields because each represented a different type of distribution, either symmetric, skewed, or bi-modal. We will refer to these three different populations as Phillips-15, Phillips-30, and Rice-15. Therefore, we had three different populations of $\mathrm{pH}$ values with 14 transects for each population and 14 points along each transect. We decided that the transects would run vertically in the grid.

Figures 1a, 2a, and 3a are histograms for the populations of Phillips-15, Phillips-30, and Rice-15, respectively and figures $1 b, 2 b$, and $3 b$ are histograms of the transect means for Phillips- 15 , Phillips-30, and Rice-15, respectively. The histograms were produced using the statistical software package SYSTAT (Berk, 1994). By examining the histograms, we can see that the Phillips-15 field is skewed to the left of the population mean, the Phillips-30 field is symmetric about the population mean, and the Rice-15 field looks symmetrically bi-modal.

\subsection{Sampling and Confidence Intervals}

Once the 14 by 14 grids of data for the three populations were established, a FORTRAN program (version 5.1 using the Watfor77 compiler, Etter, 1987) was written to perform simulations. Two separate programs were written, one to perform deterministic sampling without replacement and one to perform two-stage srswor (Sly 1996). For both sampling methods, we picked 2, 5, and 10 transects to randomly sample without replacement. We also picked 2, 7, and 14 points to be sampled on each randomly sampled transect, which is similar to what a researcher would typically use when deciding upon which locations to measure in a field study.

When executing deterministic sampling, the points along the transects were fixed instead of randomly chosen. Thus, when picking two points we chose the 5th and 10th points along each of the randomly sampled transects. For the seven points chosen, we picked the 1st, 3rd, 5th, through 13th points. Obviously, all the points along the randomly picked transects were chosen when using fourteen points.

Finally, we repeated each simulation 5000 times, and computed the proportion of times the population mean fell inside the $95 \%$ t-confidence interval. We called this proportion the capture rate of the 5000 simulations. We realize that some of the permutations in the deterministic methods are less than 5000. However, since we started with 5000 simulations in the two-stage methods we decided to keep the same number of simulations in the deterministic methods.

Two methods of estimation under deterministic sampling were considered. In the first method, we considered only the means of the $\mathrm{pH}$ values computed for each transect as the random sample. In the second method, we treated all of the sampled $\mathrm{pH}$ values as if this were a simple random sample, analogous to what researchers quite often do in field studies. Therefore, we have different standard errors for the two methods. The following are the two standard errors of the respective means for deterministic sampling using the same variable symbols which were used in the FORTRAN program: 


$$
S D E R R 1=\sqrt{\left(\frac{M-M S}{M}\right) \frac{V Y B A R}{M S}} \quad S D E R R 2=\sqrt{\left(\frac{N-K S \cdot M S}{N}\right) \frac{V A R I N D}{K S \cdot M S}}
$$

where $\mathrm{N}=$ population size $=196, \mathrm{M}=$ total number of transects $=14, \mathrm{MS}=$ number of randomly picked transects $=2,5$, or $10, \mathrm{KS}=$ number of points picked on transect $=2,7$, or 14

$$
V Y B A R=\frac{\sum_{I=1}^{m s}\left(\bar{y}_{i}-\bar{y} \cdot\right)^{2}}{M S-1} \quad V A R I N D=\frac{\sum_{i=1}^{M S} \sum_{j=1}^{K S}\left(y_{i j}-\bar{y} . .\right)^{2}}{M S \cdot K S-1}
$$

Recall that the degrees of freedom for the first and second methods of deterministic sampling using the Student t-distribution are $d f_{1}=M S-1$ and $d f_{2}=M S \cdot K S-1$, respectively. These degrees of freedom are reasonable because the first depends on the transect means sampled and the second depends on all of the points sampled.

When performing two-stage simple random sampling, we take a srswor of the transects, and then take a srswor of the points on each selected transect. We will refer to the sampling of transects as "between" sampling, and the sampling of points along the transects as "within" sampling. The simulations for the two-stage srswor were performed similarly to the deterministic sampling. Similarly, as in the deterministic sampling simulations, we chose 2,5 , and 10 transects to randomly sample and 2 and 7 locations within each transect to sample. Since all 14 points along each transect were sampled in the deterministic simulations we found it unnecessary to resimulate these for the twostage simple random sampling (that is, with 14 points, the two-stage sampling becomes single-stage sampling).

In two-stage srswor, we have variation between transects and variation within each transect. Again, we wanted to construct a $95 \%$ t-confidence interval using the two-stage standard error and the Student t-distribution. The two-stage standard error is the square root of the sum of the variance between transects and the variance within transects. The following are the variances between and within along with the two-stage standard error, where ${s_{\bar{y}}}^{2}$ is the variance of the transect means and $s_{i}{ }^{2}$ is the variance of the $i$ th transect:

$$
V A R_{B}=\left(\frac{M-M S}{M}\right) \frac{s_{\bar{y}}{ }^{2}}{M S} \quad V A R_{W}=\frac{1}{M \cdot M S}\left(\frac{K-K S}{K}\right) \sum_{i=1}^{M S} \frac{s_{i}{ }^{2}}{M S}
$$

Two-Stage Standard Error $=\sqrt{V A R_{B}+V A R_{W}}$

One problem when performing two-stage srswor was deciding what degrees of freedom to use in the t-distribution. So we decided to try three different degrees of freedom in the computations of each t-confidence interval. The first of these was the Satterthwaite degrees of freedom. To our knowledge, the Satterthwaite degrees of freedom has not been applied in computing t-confidence intervals from finite population sampling designs, except for stratified random sampling, but seemed 
like a reasonable choice. Analogous to Satterwaite's approximate df in the normal theory of components of variance in linear models (Milliken and Johnson, 1992), we constructed a finite sampling df approximation:

$$
\begin{aligned}
d f_{3}=\frac{\left[\left(\frac{M-M S}{M}\right) \frac{s_{\bar{y}}{ }^{2}}{M S}+\frac{1}{M \cdot M S}\left(\frac{K-K S}{K}\right) \sum_{i=1}^{M S} \frac{s_{i}{ }^{2}}{K S}\right]^{2}}{\left[\left(\frac{M-M S}{M}\right) \frac{s_{\bar{y}}{ }^{2}}{M S}\right]^{2}}+\sum_{i=1}^{M S} \frac{\left[\frac{1}{M \cdot M S}\left(\frac{K-K S}{K}\right) \frac{s_{i}{ }^{2}}{K S}\right]^{2}}{K S-1} \\
=\frac{\left[(M-M S) s_{\bar{y}}{ }^{2}+\left(\frac{K-K S}{K \cdot K S}\right) \sum_{i=1}^{M S} s_{i}\right]^{2}}{\left(\frac{1}{M S-1}\right)(M-M S)^{2} s_{\bar{y}}{ }^{4}+\left(\frac{1}{K S-1}\right)\left(\frac{K-K S}{K \cdot K S}\right)^{2} \sum_{i=1}^{M S} s_{i}{ }^{4}}
\end{aligned}
$$

We chose $d f_{4}=M S(K S-1)$, by analogy to split-plot designs. To compare results with $d f_{1}$, we used $d f_{5}=M S-1$. Therefore, we had five sampling strategies to consider:

$\begin{array}{ccc}\text { Sampling Strategy } & \text { Sampling Method } & \text { Degrees of Freedom } \\ 1 & \text { deterministic } & d f_{1}=M S-1 \\ 2 & \text { deterministic } & d f_{2}=M S \cdot K S-1 \\ 3 & \text { 2-stage srswor } & d f_{3} \text { (Satterthwaite) } \\ 4 & \text { 2-stage srswor } & d f_{4}=M S(K S-1) \\ 5 & \text { 2-stage srswor } & d f_{5}=M S-1\end{array}$

\section{Results and Discussion}

In this section, we will first describe the ways in which the results have been summarized. Next we will examine in detail the results of the simulations for all three fields combined, using box plots and summary statistics. For detailed results and summary statistics of each of the individual fields see Sly (1996). Finally, we will discuss two subpopulation illustrations. One is a very biased t-confidence interval estimate which resulted from the deterministic method generating a subpopulation which was extremely different from the target population. The other subpopulation we will consider is very representative of its target population, which resulted in capture rates very close to .95 . 


\subsection{Descriptions of the Summarized Results}

After running the simulations for each of the three fields, we decided to put the results for each of the five sampling strategies in a concise table. For each field, we have a three by three matrix where the rows are the number of transects selected (MS), and the columns are the number of points selected (KS) along each transect. In each cell of the matrix are the capture rates of the five sampling strategies, and the average of the lengths of the $95 \%$ t-confidence intervals for each strategy (running vertically from strategy 1-5). Tables 1,2 , and 3 contain this summary data for Phillips-15, Phillips-30, and Rice-15 fields, respectively. Table 4 is the average over all three fields for the capture rates and t-confidence interval average lengths. Note that there are blanks in each table for the two-stage srswor strategies 3,4 , and 5 where $\mathrm{KS}=14$. The two-stage simple random sampling method is not appropriate when sampling all of the points along a transect. These capture rates should be similar to the deterministic methods 1 and 2 , since all fourteen points are being chosen along each randomly selected transect and only the degrees of freedom may be different. Note that the degrees of freedom for strategy 3 would change only through the variance of means (across transects) for a given MS.

After running all of the simulations, we constructed five side by side box plots of each sampling strategy, using the capture rates from all three fields combined. The box plots of the capture rates combining all three fields may be referenced in Figure 4. We also collapsed the capture rates over each field. Figures 5, 6, and 7 are five side by side box plots, one for each sampling strategy over the fields Phillips-15, Phillips-30 and Rice-15, respectively. The number of capture rates going into each box plot is denoted by $\mathrm{n}$, and is located next to the lower right corner of each box plot. Recall that the confidence level of the t-confidence intervals was 95 percent. Therefore, lines were drawn at the 95 percent capture rate level on figures $4,5,6$, and 7 .

\subsection{Results of All Simulations}

We will consider Figure 4, since the capture rates from all three fields combined give an overall view of the simulation results. Our basis of interpretation is that a researcher would know the sampling strategy to be used, but not know the characteristics of the field data. The differences between the five box plots will be at least one of the following: 1) the degrees of freedom; 2) the standard error; and 3) the fact that strategies 1 and 2 use possibly biased samples when estimating the population mean (i.e., the expected value of the sample mean for strategies 1 and 2 is not equal to the population mean). The reason that strategies 1 and 2 have possibly biased samples is that they are sampling subpopulations (i.e., they are sampling a subset of the 14 by 14 population grid). Note that the expected value of the sample mean is equal to the population mean for the two-stage sampling strategies 3, 4, and 5 .

When looking at Figure 4 we immediately see that the first two sampling strategies, which used the deterministic method of sampling, are extremely different. Recall that strategy 1 used the transect means as the random sample whereas strategy 2 used all of the individual sample values as the random sample. Most importantly, the variation of capture rates is almost four times as large in strategy 2 as compared to strategy 1 . This difference in variation is because the margin of error of strategy 2 is smaller than the margin of error for strategy 1. Descriptive statistics of the capture rates for the five sampling strategies are summarized in Table 5. From Table 5, we see that the standard 
deviation of the strategy 2 capture rates is twice as large as that of strategy 1 . The median of the capture rates for strategy 1 is approximately .94 , whereas the median for strategy 2 is approximately .85. The minimum capture rate of strategy 1 is .654, but the minimum capture rate of strategy 2 is .451. Also, the maximum capture rate of strategy 1 is .978 and the maximum capture rate of strategy 2 is .996. Descriptive statistics for the average length of the 5000 simulated t-confidence intervals for each of the five sampling strategies may be referenced in Table 6. From Table 6, we see that the average length of the t-confidence intervals over all three fields is 1.897 for strategy 1 and only 0.462 for strategy 2. So the t-confidence intervals are too small in general for strategy 2, which causes the capture rate to be extremely low. Thus, in general when doing deterministic sampling we would recommend using sampling strategy 1 over sampling strategy 2 , which treats the transects as the random sample instead of the locations as the random sample. This is consistent with the randomization scheme of selecting transects, but not locations on transects.

One can immediately notice when looking at the Figure 4 box plots that the two-stage simple random sampling strategies 3, 4, and 5 have a smaller variation (using IQR's) in capture rates than the deterministic sampling strategies 1 and 2. The IQR's of the box plots of the capture rates of sampling strategies 3 and 4 are below the $95 \%$ capture rate line. However, the box plot for the strategy 5 capture rates is entirely above the 95 percent capture rate line. This means that the $t-$ confidence intervals using sampling strategy 5 are wider than they "should be" (i.e. the t-intervals are conservative). We would also like to note that both deterministic box plots have IQR's which are below the 95 percent capture rate line similar to strategies 3 and 4. This is because the deterministic strategies sample subpopulations. Therefore, the deterministic strategies would tend to have biased tconfidence intervals of the population mean at 95 percent confidence, which means that the capture rates are smaller than they "should be". We recommend in general to perform the two-stage simple random sampling strategies instead of the deterministic sampling strategies, since their capture rates are closer in general to the $95 \%$ capture rate line and have smaller variation. We do not recommend strategy 2 because it has a smaller, inappropriate margin of error and it is potentially biased.

Now we will compare the three two-stage simple random sampling strategies, which are strategies 3, 4, and 5. When looking at the Figure 4 box plots, one can easily see that strategy 4 has the largest variation of capture rates, strategy 5 has the smallest variation, and strategy 3 has variation between strategies 4 and 5 . In fact, when examining Table 5 we see that strategy 3 has a standard deviation of capture rates .01 larger than strategy 5. Also note that strategy 4 has a standard deviation of capture rates approximately twice as large as the standard deviation of strategy 3 capture rates. As far as recommending a strategy to use, one would tend to argue for strategy 3 or 5 . We recommend using strategy 3 because its median capture rate is .945 , which is closer to the 95 percent capture rate than the median capture rate of strategy 5 which is .964 . From the box plots, we can also see that the variation of capture rates for strategy 3 is acceptable. Furthermore, strategy 5 seems to be too conservative in its capture rates. By examining Table 6, we see that the average confidence interval length is 2.201 for strategy 5 and only 1.843 for strategy 3 .

Some might argue that strategy 5 is the best to apply because it is conservative in its capture rate when compared to the other sampling strategies, (it captures the population mean almost always), and it has the smallest variation in capture rates. Recall that the degrees of freedom is the only difference between strategies 3 and 5. We used the Satterthwaite degrees of freedom for strategy 3 , and the number of transects minus one degrees of freedom for strategy 5 . In most cases the degrees of freedom for strategy 3 is a little larger than the degrees of freedom for strategy 5 . When the 
degrees of freedom is smaller, the t-critical value for the t-confidence interval is larger. Therefore, since the degrees of freedom for strategy 5 are smaller than strategy 3 , its t-confidence intervals are larger. Thus, another reason we recommend using strategy 3 is that the 95 percent confidence interval for strategy 5 is probably too large in some cases. This fact may be examined by looking at Table 4. The averages over all three fields of the t-confidence interval lengths for strategy 3 are smaller than that of strategy 5 in general, except for $(K S=2, M S=5)$ and $(K S=2, M S=10)$.

Again, consider the Figure 4 box plots. When looking at the deterministic methods (strategies 1 and 2), we see only one outside value denoted by an asterisk in strategy 1, and we will illustrate below why this value is so low.

\subsection{Subpopulation Illustration}

Consider the box plot of capture rates for deterministic strategy 1 in Figure 4, and note that there is only one outside value denoted by an asterisk. The capture rate of this outside value is only .654 , and it is from the Phillips-30 field when $\mathrm{MS}=10$ and $\mathrm{KS}=2$. We would also like to point out that the next lowest capture rate of .794 for strategy 1 is again from the Phillips-30 field when $\mathrm{MS}=5$ and $\mathrm{KS}=2$. Recall that we chose the 5 th and 10th locations to sample along the randomly selected transects when sampling two points deterministically $(\mathrm{KS}=2)$. We decided to calculate $y_{i j}-\mu$ for the entire field, where $\mu=5.838$ is the population mean $\mathrm{pH}$ value and $y_{i j}$ is the $j$ th location on the $i$ th transect. The computed values of $y_{i j}-\mu$ on locations five and ten turn out to be mostly negative, with ten out of fourteen and twelve out of fourteen negative differences, respectively. Also note that the subpopulation mean and subpopulation standard deviation are $\mu_{s u b}=5.73$ and $\sigma_{s u b}=.36$, respectively. However, the population mean is $\mu=5.838$ and the population standard deviation is $\sigma=.48$. Therefore, when randomly sampling ten transects and deterministically picking the 5th and 10th locations on this field, the average of the transect means usually underestimated the population mean and the t-confidence intervals are smaller because of the smaller standard deviation. Thus, this subpopulation is not representative of the entire population for the Phillips-30 field and the t-confidence intervals do a poor job of capturing the population mean.

Consider the Rice-15 field when deterministically choosing the fifth and tenth locations (KS $=2$ ) for an illustration of a subpopulation which is very representative of the entire population. Note that the capture rates for strategy 1 are $.926, .952$, and .970 for $\mathrm{MS}=2$, MS $=5$, and $\mathrm{MS}=10$, respectively. These are very close to the target capture rate of .950 . The population mean and the population standard deviation for the Rice- 15 field are $\mu=6.09$ and $\sigma=.63$, respectively. On the other hand, the subpopulation mean and subpopulation standard deviation for the fifth and tenth locations are $\mu_{s u b}=6.11$ and $\sigma_{s u b}=.64$, respectively. Since these parameters are so close to each other, this helps explain why the average of the transect means usually is very close to the population mean and why the t-confidence intervals capture the population mean so well for this particular subpopulation. 


\section{Summary}

In most instances a researcher in agriculture does not know the characteristics of the field to be sampled. When considering our simulation results, we recommend the two-stage srswor method, and, if constructing t-confidence interval estimates, the Satterthwaite degrees of freedom (strategy 3). In general, using the deterministic method of transect sampling may produce estimates that are very biased, so we discourage the use of the deterministic method. However, there are cases in which a researcher will prefer to sample a field deterministically after randomly selecting transects. In addition to sampling deterministically, a researcher will quite often treat the individual locations as the random sample instead of the transect means as the random sample. In light of our simulation results, we recommend sampling strategy 1 over strategy 2 if deterministic sampling must be performed and the characteristics of the field are unknown. Recall that Strategy 1 and strategy 2 both use the deterministic method of sampling, but strategy 1 treats the transect means as the random sample whereas strategy 2 treats the locations as the random sample.

Further investigation of transect sampling for these particular data sets has been considered.

One idea is to try a repeated systematic approach to sampling within transects. It is reasonable to assume that the capture rates for systematic strategies would be between the deterministic and twostage sampling strategies capture rates. Another topic which could be investigated would be to generate a field containing normally distributed values. In this case we would want the transects to be normally distributed with the same mean and variance along each transect so that we could directly compare deterministic strategies 1 and 2. Alternatively, we could construct a large data set so that we could take a large sample and use the Central Limit Theorem.

\section{References}

Berk, K.N., 1994, Data Analysis with Student Systat, Course Technology, Inc.: United States.

Cochran, W.G., 1977, Sampling Techniques, 3rd edition, John Wiley: New York.

Etter, D.M. 1987, Structured FORTRAN 77 for Engineers and Scientists, Benjamin/Cummings: Menlo Park, California.

Milliken, G.A. and Johnson, D.E., 1992, Analysis of Messy Data, Volume I: Designed Experiments, Chapman and Hall: New York and London.

SAS, 1985, SAS/GRAPH User`s Guide, Version 5 edition, SAS Institute Inc.: Cary, NC.

Satterthwaite, F.E., 1946, An Approximate Distribution of Estimates of Variance Components, Biometrics Bulletin, 2: 110-114.

Sly, S. N. 1996, Validity of 95\% t-confidence Intervals Under Some Transect Sampling Strategies, M.S. Thesis, Department of Statistics, Kansas State University, Manhattan KS. 


\section{Phillips - 15}

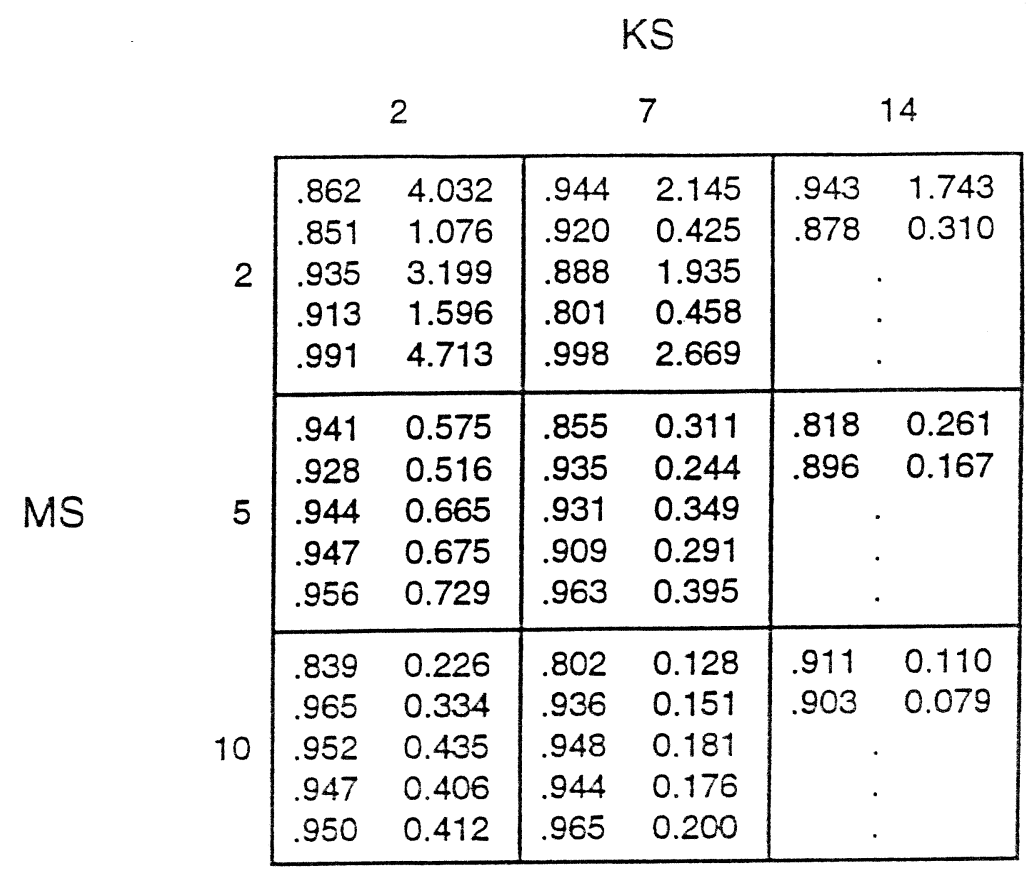

Table 1 Capture rates of $\mu$ for $K S=$ number of points sampled along each transect, and MS = number of transects randomly sampled. The five capture rates in each cell represent the capture rates under the sampling strategies $1,2,3,4$, and 5 , respectively from top to bottom using the Phillips-15 field. The second column in each cell is the average length of the 5000 confidence intervals created for each of the five sampling strategies, respectively.

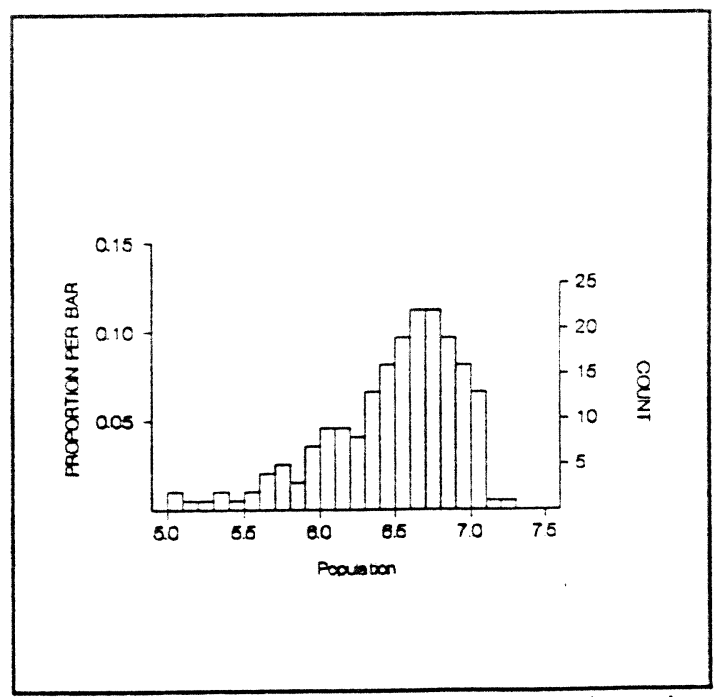

Figure 1a Histogram of the population of $\mathrm{pH}$ values for the Phillips -15 field.

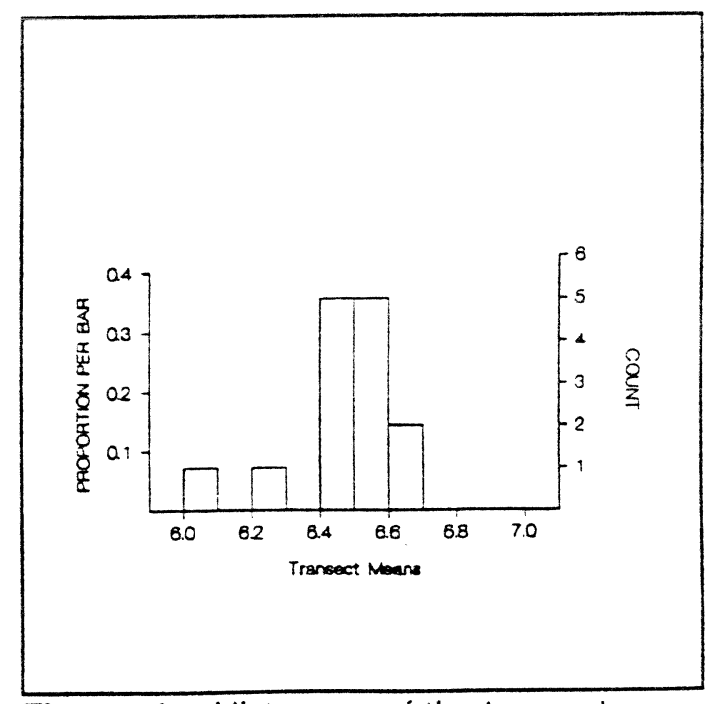

Figure $1 \mathrm{~b}$ Histogram of the transect means of $\mathrm{pH}$ values for the Phillips-15 field. 


\section{Phillips -30}

\begin{tabular}{|c|c|ll|ll|}
\multicolumn{2}{c}{2} & \multicolumn{2}{c}{ KS } \\
\hline \multicolumn{1}{c}{ MS } & \multicolumn{1}{c}{7} \\
\hline .926 & 4.682 & .978 & 3.385 & .953 & 3.450 \\
.828 & 1.189 & .855 & 0.520 & .595 & 0.326 \\
.939 & 4.153 & .891 & 3.296 & &. \\
.916 & 1.911 & .763 & 0.676 & &. \\
.993 & 5.645 & .992 & 3.945 & &. \\
\hline .794 & 0.656 & .960 & 0.475 & .940 & 0.485 \\
.818 & 0.576 & .853 & 0.300 & .653 & 0.181 \\
.949 & 0.761 & .938 & 0.523 & &. \\
.953 & 0.772 & .902 & 0.412 & &. \\
.961 & 0.834 & .959 & 0.561 & &. \\
\hline .654 & 0.266 & .885 & 0.188 & .942 & 0.192 \\
.846 & 0.379 & .867 & 0.185 & .653 & 0.085 \\
.958 & 0.442 & .951 & 0.235 & &. \\
.952 & 0.433 & .942 & 0.223 & &. \\
.959 & 0.440 & .967 & 0.253 & &. \\
\hline
\end{tabular}

Table 2 Capture rates of $\mu$ for $\mathrm{KS}=$ number of points sampled along each transect, and MS = number of transects randomly sampled. The five capture rates in each cell represent the capture rates under the sampling strategies $1,2,3,4$, and 5 , respectively from top to bottom using the Phillips-30 field. The second column in each cell is the average length of the 5000 confidence intervals created for each of the sampling strategies, respectively.

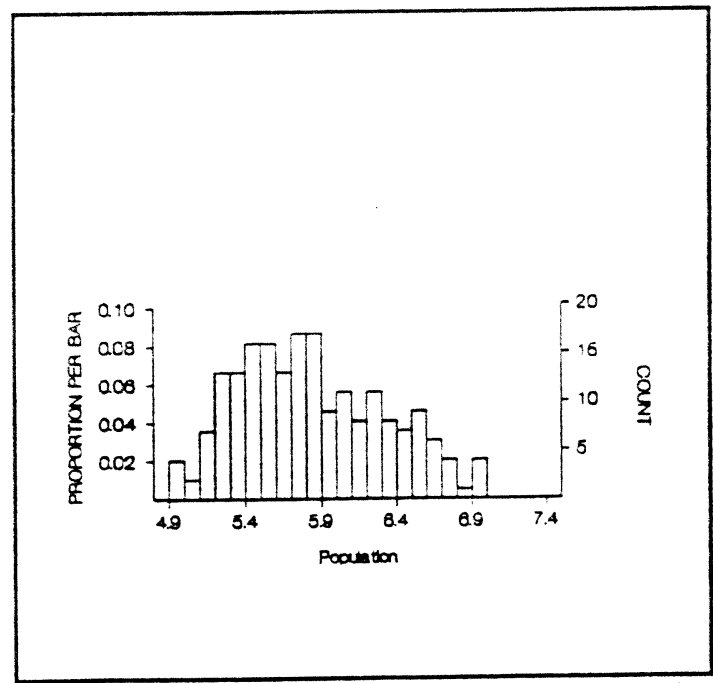

Figure 2a Histogram of the population of $\mathrm{pH}$ values for the Phillips-30 field.

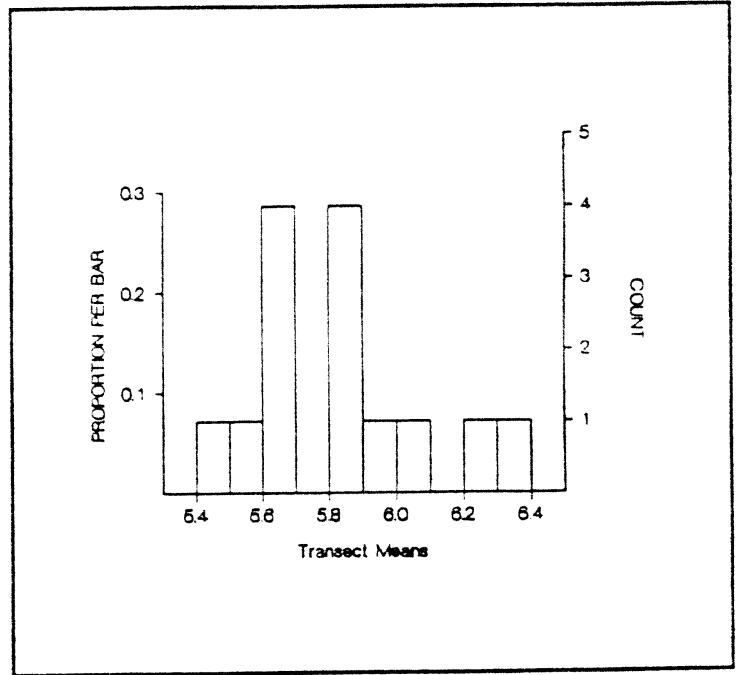

Figure $2 b$ Histogram of the transect means of $\mathrm{pH}$ values for the Phillips-30 field. 


\section{Rice -15}

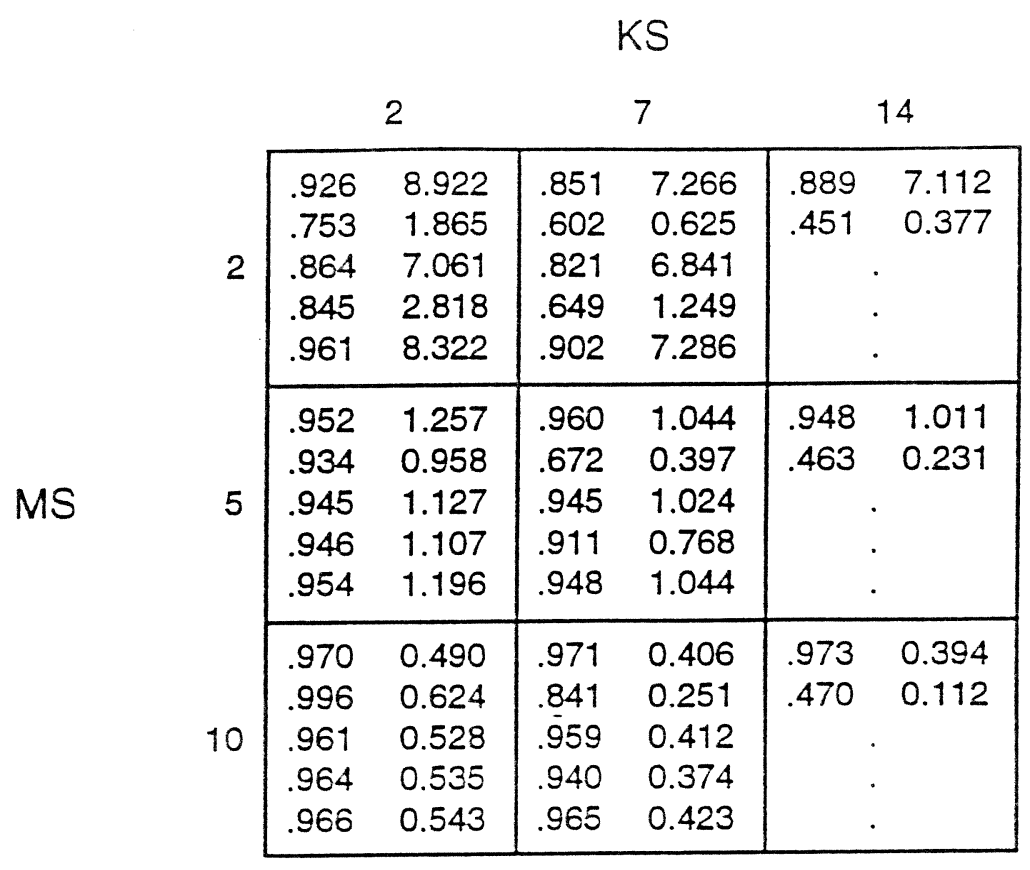

Table 3 Capture rates of $\mu$ for $\mathrm{KS}=$ number of points sampled along each transect, and $M S=$ number of transects randomly sampled. The five capture rates in each cell represent the capture rates under the sampling strategies $1,2,3,4$, and 5 , respectively from top to bottom using the Rice-15 field. The second column in each cell represents the average length of the 5000 confidence intervals created for each of the sampling strategies, respectively.

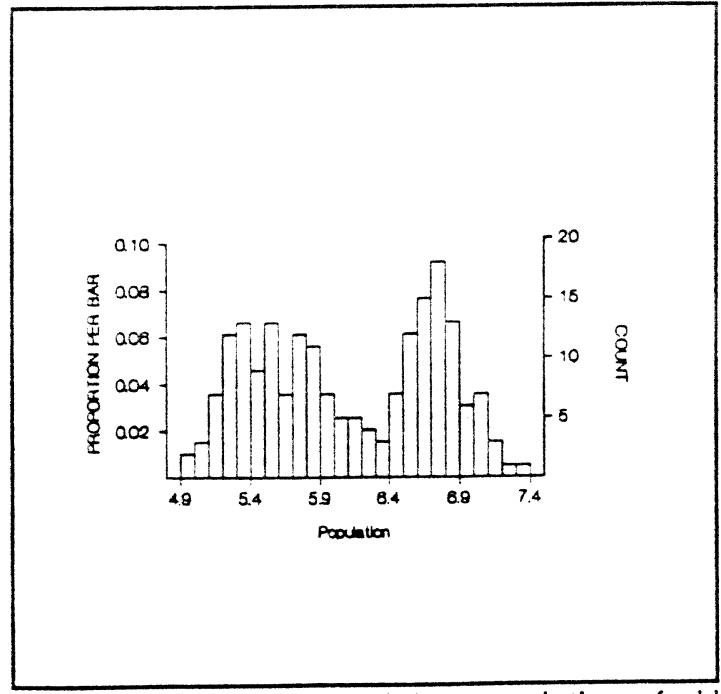

Figure 3a Histogram of the population of $\mathrm{pH}$ values for the Rice-15 field.

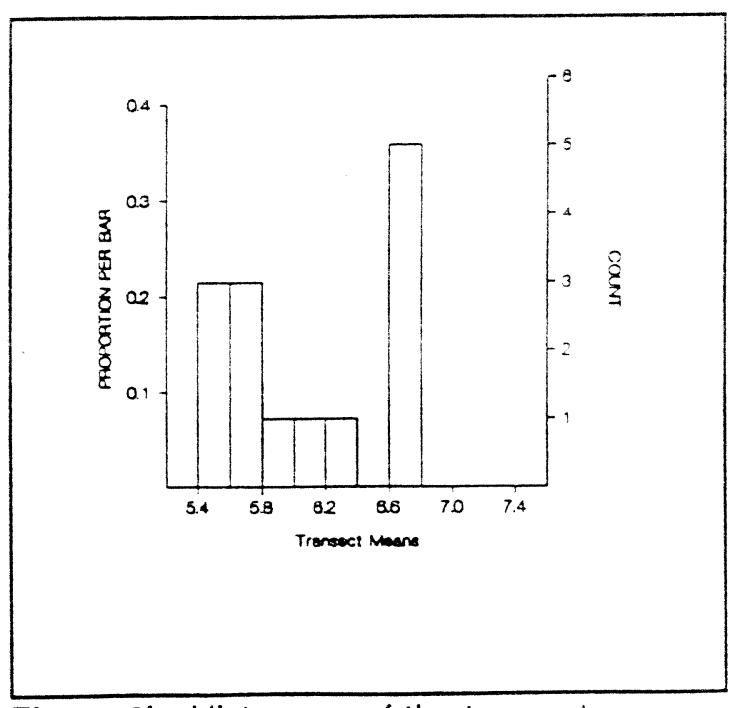

Figure 3b Histogram of the transect means of $\mathrm{pH}$ values for the Rice-15 field. 


\section{All Fields}

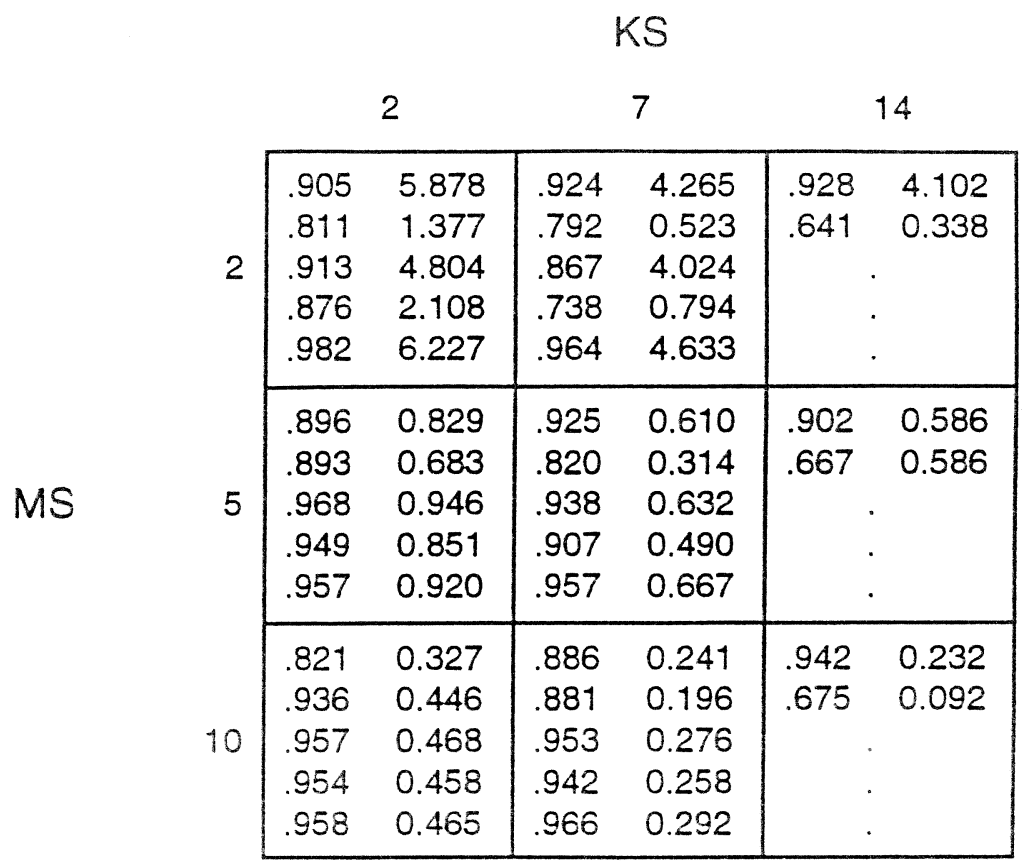

Table 4 Capture rates of $\mu$ for $K S=$ number of points sampled along each transect, and $M S=$ number of transects randomly sampled. The five capture rates in each cell represent the average capture rates over all three fields under the sampling strategies 1, 2, 3, 4, and 5 , respectively. The second column in each cell is the average length over all three fields of the average length of the 5000 confidence intervals created for each of the five sampling strategies, respectively.

(All Fields) Capture Rates

Sampling Strategy

\begin{tabular}{lccccc} 
& 1 & 2 & 3 & 4 & 5 \\
\hline Mean & .903 & .791 & .929 & .894 & .964 \\
Median & .940 & .851 & .945 & .928 & .962 \\
Std. Dev. & .075 & .161 & .038 & .085 & .028 \\
Min & .654 & .451 & .821 & .649 & .902 \\
Max & .978 & .996 & .961 & .964 & .998 \\
n & 27 & 27 & 18 & 18 & 18
\end{tabular}

Table 5 Sample statistics for the capture rates of the five different sampling strategies combining all the fields.
(All Fields) Average C.I. Length

Sampling Strategy

\begin{tabular}{lccccc} 
& 1 & 2 & 3 & 4 & 5 \\
\hline Mean & 1.897 & 0.462 & 1.843 & 0.827 & 2.201 \\
Median & 0.575 & 0.334 & 0.713 & 0.605 & 0.782 \\
Std. Dev. & 2.488 & 0.402 & 2.200 & 0.688 & 2.625 \\
Min & 0.110 & 0.079 & 0.181 & 0.176 & 0.200 \\
Max & 8.922 & 1.865 & 7.061 & 2.818 & 8.322 \\
n & 27 & 27 & 18 & 18 & 18
\end{tabular}

Table 6 Sample statistics for the average of the confidence Interval lengths of the five sampling strategies combining all the fields. 


\section{All Fields}

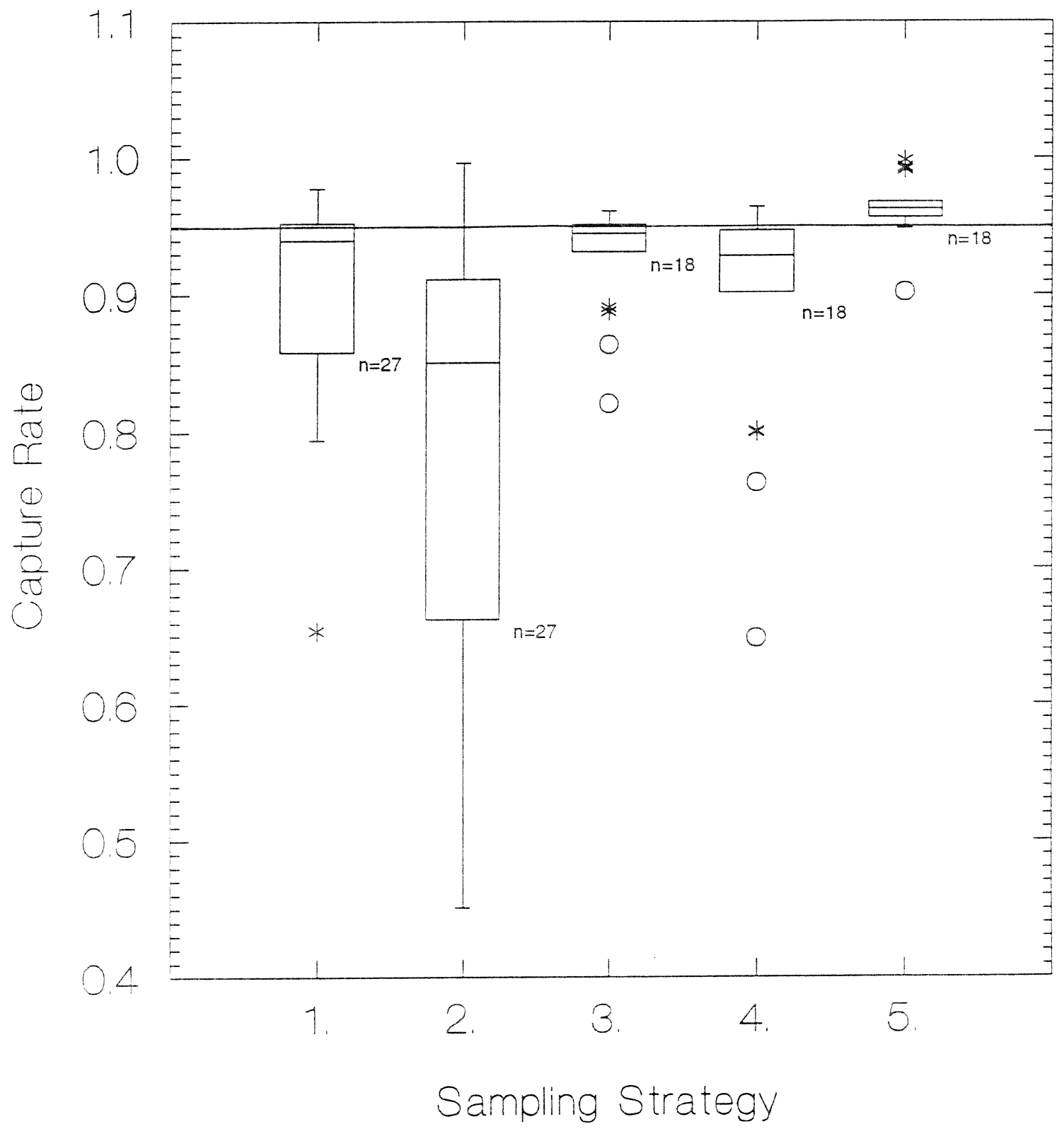

Figure 4 Box plots of capture rates for the five sampling strategies using all three fields. 


\section{Phillips-15}

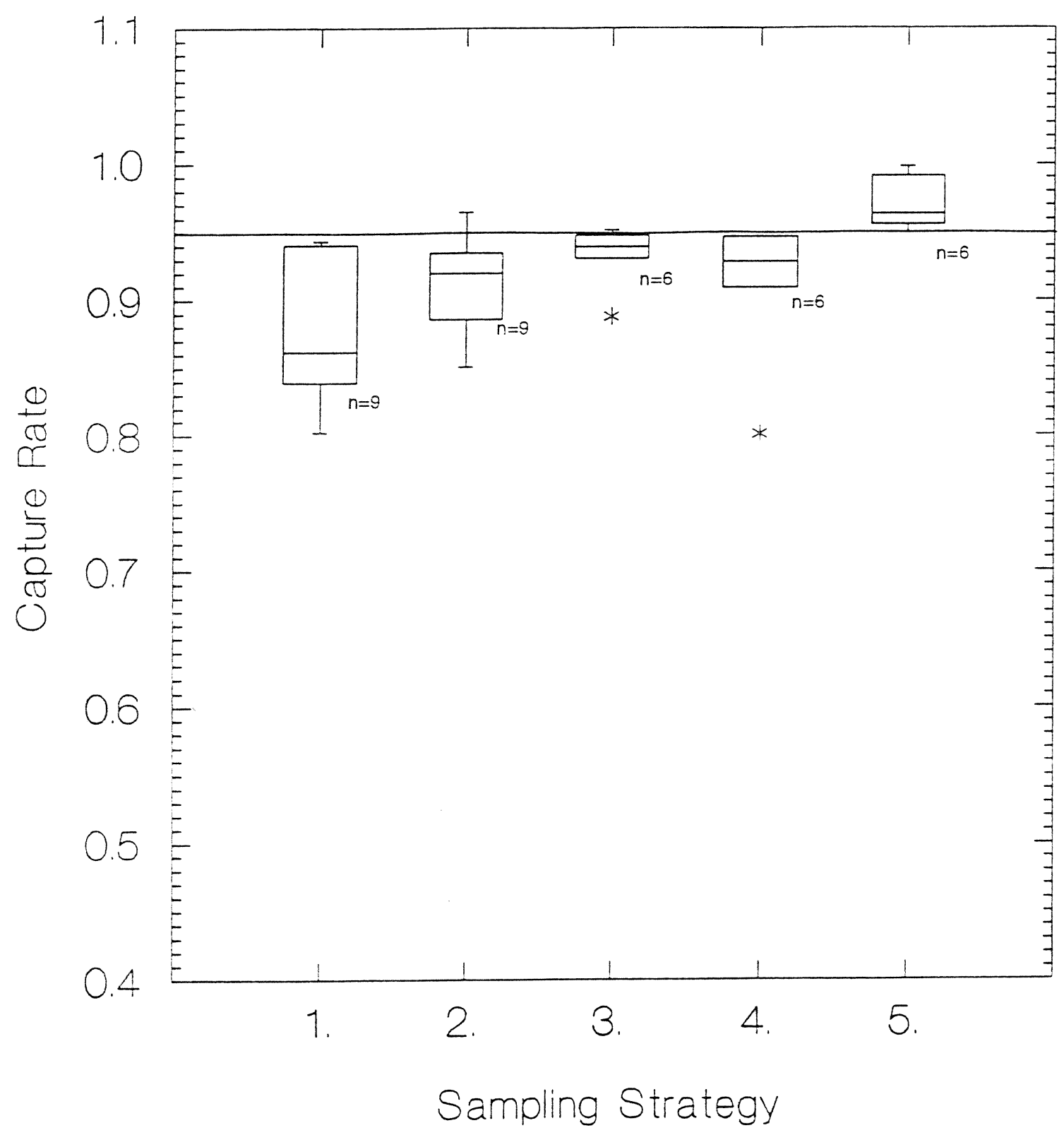

Figure 5 Box plots of capture rates for the five sampling strategies using the Phillips-15 data. 


\section{Phillips-30}

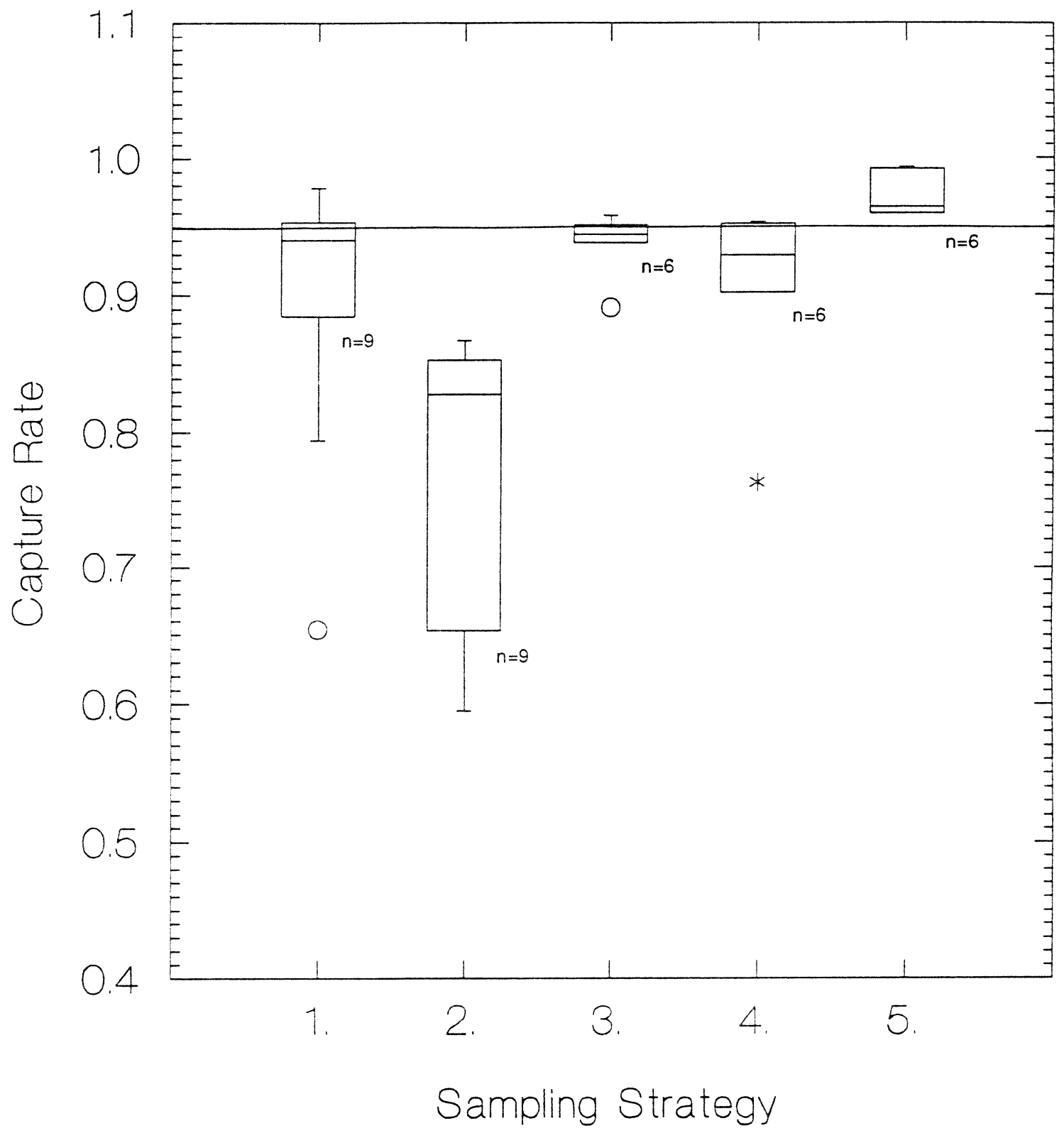

Figure 6 Box plots of capture rates for the five sampling strategies using the Phillips-30 data. 


\section{Rice-15}

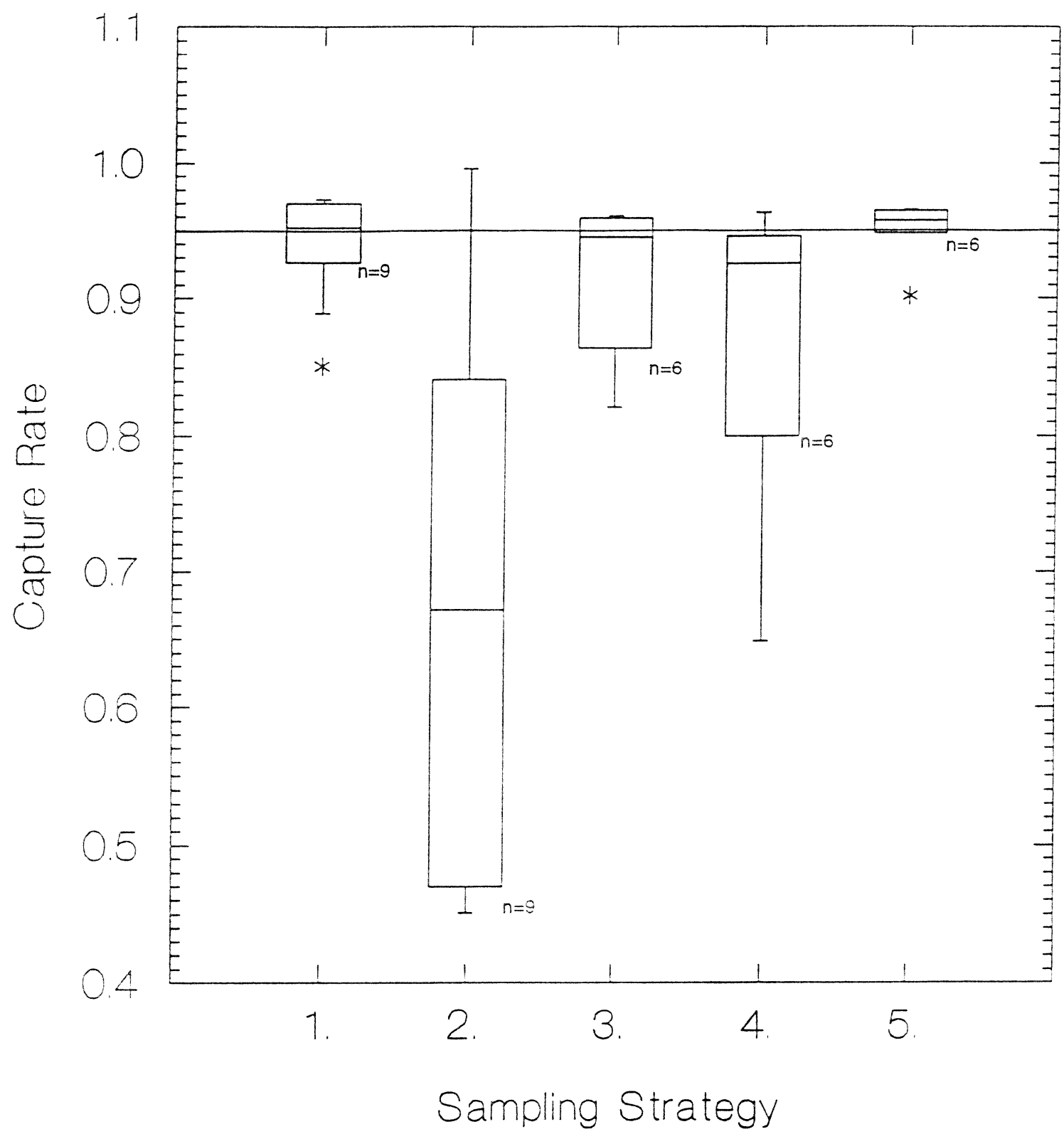

Figure 7 Box plots of the capture rates for the five sampling strategies using the Rice-15 data. 